\title{
The afterglow of GRB 050709 and the nature of the short-hard $\gamma$-ray bursts
}

\author{
D. B. Fox ${ }^{1,2}$ D. A. Frail,${ }^{3}$ P. A. Price,${ }^{4}$ S. R. Kulkarni, ${ }^{1}$ E. Berger, ${ }^{5}$ T. Piran, ${ }^{1,6}$ A. M. Soderberg, ${ }^{1}$ \\ S. B. Cenko, ${ }^{1}$ P. B. Cameron, ${ }^{1}$ A. Gal-Yam,${ }^{1}$ M. M. Kasliwal,${ }^{1}$ D.-S. Moon, ${ }^{1}$ F. A. Harrison, ${ }^{1}$ \\ E. Nakar, ${ }^{1}$ B. P. Schmidt,${ }^{7}$, B. Penprase,${ }^{8}$ R. A. Chevalier,${ }^{9}$ P. Kumar, ${ }^{10}$ K. Roth ${ }^{11}$, D. Watson, ${ }^{12}$ \\ B. L. Lee, ${ }^{13}$ S. Shectman, ${ }^{5}$ M. M. Phillips,${ }^{5}$ M. Roth,${ }^{5}$ P. J. McCarthy,${ }^{5}$ M. Rauch,${ }^{5}$ L. Cowie,${ }^{4}$ \\ B. A. Peterson, ${ }^{7}$ J. Rich, ${ }^{7}$ N. Kawai, ${ }^{14}$ K. Aoki, ${ }^{15}$ G. Kosugi, ${ }^{15}$ T. Totani, ${ }^{16}$ H.-S. Park, ${ }^{17}$
}

A. MacFadyen ${ }^{18} \&$ K. C. Hurley ${ }^{19}$

${ }^{1}$ Division of Physics, Mathematics \& Astronomy, California Institute of Technology, Pasadena, CA 91125, USA

2 Department of Astronomy \& Astrophysics, 525 Davey Laboratory, Pennsylvania State University, University Park, PA 16802, USA

3 National Radio Astronomy Observatory, P.O. Box O, Socorro, NM 87801, USA

${ }^{4}$ University of Hawaii, Institute for Astronomy, 2680 Woodlawn Drive, Honolulu, HI 96822, USA

${ }^{5}$ Carnegie Observatories, 813 Santa Barbara Street, Pasadena, CA 91101, USA

${ }^{6}$ Racah Institute for Physics, The Hebrew University, Jerusalem 91904, Israel

7 Research School of Astronomy and Astrophysics, The Australian National University, Weston Creek, ACT 2611, Australia

8 Pomona College, 610 North College Avenue, Claremont, CA 91711, USA

9 Department of Astronomy, University of Virginia, P.O. Box 3818, Charlottesville, VA 22903, USA

10 Astronomy Department, University of Texas, Austin, TX 78731, USA

11 Gemini Observatory, 670 North A'ohoku Place, Hilo, HI 97620, USA

12 Niels Bohr Institute, University of Copenhagen, Juliane Maries Vej 30, DK-2100 Copenhagen, Denmark

13 Department of Astronomy \& Astrophysics, University of Toronto, Toronto, Ontario, M5S 3H8, Canada

14 Department of Physics, Tokyo Institute of Technology, Ookayama 2-12-1, Meguro-ku, Tokyo 152-8551, Japan

15 Subaru Telescope, National Astronomical Observatory of Japan, 650 N. A'ohoku Place, Hilo, Hawaii 76720, USA

${ }^{16}$ Department of Astronomy, School of Science, Kyoto University, Sakyo-ku, Kyoto 606-8502, Japan

17 Lawrence Livermore National Laboratory, 7000 East Avenue, Livermore, CA 94550, USA

18 Institute for Advanced Study, Princeton, NJ 08540, USA

19 Space Sciences Laboratory, University of California, Berkeley, CA 94720, USA

The final chapter in the long-standing mystery of the gamma-ray bursts (GRBs) centres on the origin of the short-hard class, suspected on theoretical grounds to result from the coalescence of neutron star or black hole binary systems. Numerous searches for the afterglows of short-hard bursts have been made, galvanized by the revolution in our understanding of long-duration GRBs that followed the discovery in 1997 of their broadband (X-ray, optical, and radio) afterglow emission. Here we present the 
2 Fox et al.

discovery of the X-ray afterglow of a short-hard burst whose accurate position allows us to unambiguously associate it with a star-forming galaxy at redshift $z=0.160$, and whose optical lightcurve definitively excludes a supernova association. Together with results from three other recent short-hard bursts, this suggests that short-hard bursts release much less energy than the long-duration GRBs. Models requiring young stellar populations, such as magnetars and collapsars, are ruled out, while coalescing degenerate binaries remain the most promising progenitor candidates.

\section{Introduction}

High-energy transients remain at the frontier of astrophysics research because they probe extreme physical regimes of matter, gravity, and energy density. Soft $\gamma$-ray repeaters (SGRs) combine matter at supra-nuclear densities with magnetic fields in excess of $10^{15} \mathrm{G}$, while long-duration GRBs, which probably herald the birth of stellar-mass black holes, drive ultra-relativistic outflows and power the brightest explosions in the Universe. Progress in understanding new classes of high-energy transient has typically required a multiwavelength approach; in particular, the identification of longer-wavelength counterparts enables precision localization and detailed studies. It was the discovery of the slow-fading 'afterglow' emission of long-duration GRBs that enabled their sub-arcsecond localization, the measurement of their redshifts, the identification of their star-forming host galaxies, the quantification of their energy scale, and ultimately, established their connection to the deaths of massive stars (see ref 1 for a review).

The nature of the short-hard gamma-ray bursts (SHBs) has been an outstanding mystery of high-energy astrophysics for more than 30 years. The SHBs comprise about $30 \%$ of the GRB population at the Burst and Transient Source Experiment (BATSE) threshold and have typical durations of $0.3 \mathrm{~s}$ and peak energies of order $350 \mathrm{keV}$, with power-law tails extending to higher energies. ${ }^{2}$ Despite extensive searches, ${ }^{3}$ no short-hard burst has yet been sufficiently well-localized to ascertain its origins. Historically, $\gamma$-ray satellites were either not sensitive to SHBs or provided positions that were too crude or too delayed to enable deep searches. A significant breakthrough came when the X-ray telescope (XRT) on the recently-launched 
Swift satellite detected the rapidly-fading afterglow of GRB 050509B and localized it to a circular region of radius 9.3 arcseconds. Within this X-ray localization there are nearly 50 objects identified in Hubble Space Telescope (HST) images, ${ }^{4}$ the brightest of which by far is an elliptical galaxy at $z=0.2248$ that has been proposed as the likely host galaxy of this burst. ${ }^{5,6}$

Even without any SHB distance determinations, the isotropic sky distribution and noneuclidean brightness distribution of the SHBs suggest a cosmological origin, ${ }^{2,7}$ fuelling speculation that short-hard bursts are the result of the coalescence of compact object (neutron star-neutron star or black hole-black hole) binaries. ${ }^{8}$ Theoretical estimates yield merger rates $^{9}$ that can easily accommodate the observed burst rate, with engine lifetimes and energy releases roughly consistent with the burst properties for a cosmological population. Nonetheless, without any detailed knowledge of their distances, energetics, and environments, younger progenitor populations such as magnetars and collapsars cannot be ruled out. If the coalescence model is correct, the SHBs will be a primary source population for the Laser Interferometer Gravitational Wave Observatory and other ground-based gravitational wave detectors. As such, the SHBs promise to provide a crucial test-bed for theories of strong-field gravity, the nuclear equation of state and the formation of black holes.

\section{Discovery of the X-ray afterglow}

Upon receiving notification of the localization ${ }^{10}$ of the short-hard burst GRB 050709 by the High-Energy Transient Explorer (HETE), we initiated observations with the Chandra X-ray Observatory as part of our approved program for the SHBs, observing the 81-arcsec error

circle with the Advanced CCD Imaging Spectrometer (ACIS). ${ }^{11}$ A total of $38.4 \mathrm{ks}$ of good data were obtained after excluding intervals of background flaring activity, at a mean epoch of 2.52 days after the burst (Table 1). We detected two sources in the HETE error circle: a faint and resolved (or double) source and a bright point source (see Figure 1). The faint source is well detected at low energies (0.3-2.0 keV band), has a flux of $3.0 \times 10^{-15} \mathrm{erg} \mathrm{cm}^{-2} \mathrm{~s}^{-1}(1-5$ $\mathrm{keV}$ band) and coincides with a catalogued radio source from the National Radio Astronomy Observatory Very Large Array (VLA) 20-cm Sky Survey ${ }^{12}$ (NVSS). 
The bright point source has $49.5 \pm 8.8$ counts in the $0.3-8 \mathrm{keV}$ band, corresponding to an X-ray flux of $3.5 \times 10^{-15} \mathrm{erg} \mathrm{cm}^{-2} \mathrm{~s}^{-1}$ (1-5 keV band) for the best-fit power-law spectrum (photon index $\alpha=2.24 \pm 0.35$, with column density fixed to the expected Galactic hydrogen column density, $\left.N_{\mathrm{H}}=1.2 \times 10^{20} \mathrm{~cm}^{-2}\right)$. The source is located at $\alpha=23: 01: 26.96, \delta=$ -38:58:39.5 (J2000). This position has been corrected by 0.4 arcsecond from the native Chandra astrometry using three X-ray sources coincident with stars in the United States Naval Observatory (USNO) B1.0 catalog. We estimate the $90 \%$ confidence radius is 0.5 arcsecond.

We proposed ${ }^{13}$ the brighter source as the X-ray afterglow of GRB 050709. We also noted that it was offset by about one arcsecond from a faint $(R \sim 20.5 \mathrm{mag})$ source visible in the Digitized Sky Survey, plausibly its host galaxy. Making use of the Chandra position, we find a marginal detection of the X-ray source in earlier observations made by the Swift XRT (Table 1).

We then executed an 18-ks follow-up observation (mean epoch 16.0 days post-burst) with an identical observatory configuration. These data showed that, at a $99.7 \%$ confidence level, the X-ray source had faded, roughly by a factor of two. Inspecting the eleven events within a 1.5-arcsec radius of the X-ray afterglow position, we found that nine events occurred within the first third of the observation. This is in contrast to the first epoch, which exhibits a roughly uniform count rate over the observation. A Kolmogorov-Smirnov (KS) test demonstrates that the second epoch arrival times are inconsistent with a steady event rate at $99.9 \%$ confidence. We therefore suggest that during the first $\sim 6 \mathrm{ks}$ of this observation the source was in a "flaring" state, roughly an order of magnitude brighter than during the remainder of the observation.

In Table 1 we give the mean epochs and X-ray fluxes for the flaring and quiescent portions of this observation. Using the quiescent flux, which represents only a marginal detection of emission (90\% confidence), we find a temporal decay index in the X-ray band of $\alpha_{\mathrm{X}} \lesssim-1$ for the interval from 2.5 to 16 days after the burst.

The discovery of this flaring behavior suggests that even 16 days after the burst, the afterglow is still subject to new energy inputs. The sudden cessation of the flare represents 
a small fraction of the time since the burst, indicating that the flaring must arise from a source physically distinct from the fading afterglow. We suggest that the flare arises from ongoing activity of the central engine, in analogy to the bright X-ray flares observed from several long-duration Swift GRBs. ${ }^{14}$

\section{Optical afterglow and host galaxy}

In addition to our Chandra observations we conducted an extensive ground-based campaign on GRB 050709 at radio, optical and near-infrared wavelengths using the VLA, the 40-inch Swope and 100-inch Du Pont telescopes at Las Campanas Observatory, and the 8.2-m Subaru Telescope on Mauna Kea. A complete list of these observations is given in Table 1, along with upper limits on the flux of the afterglow at these epochs and measurements of the host galaxy brightness.

Our Chandra afterglow candidate was found to be coincident with a point-like optical source, ${ }^{13}$ distinct from the candidate host galaxy, which faded in a manner consistent with the optical afterglows of long-duration GRBs. ${ }^{15}$ We undertook spectroscopy of the candidate host galaxy with the Gemini Multi-Object Spectrograph on the Gemini North telescope, and find it to be a star-forming galaxy at redshift $z=0.160$ (Fig. 2).

We also triggered a sequence of HST observations with the Advanced Camera for Surveys $^{16}$ (ACS). Within the Chandra error circle we find a single bright, fading, point-like source, the unambiguous optical afterglow of GRB 050709; our HST photometry is presented in Table 1. Expressing the afterglow decay as a power-law (flux $\propto t^{\alpha}$ ) between each epoch, we find power-law indices of $\alpha_{12}=-1.25 \pm 0.09$ between epochs 1 and $2, \alpha_{23}=-2.83 \pm 0.39$ between epochs 2 and 3 , and $\alpha_{34}<-0.43$ between epochs 3 and 4 . Our observation of a break in the decay is definitive; the HST data are inconsistent with a single power-law decay at the 3.7- $\sigma$ level.

As can be seen from Figure 1, the optical afterglow of GRB 050709 is superposed on the outskirts of the $z=0.16$ candidate host galaxy. This precise localization, the first subarcsecond localization for any short-hard burst, unambiguously associates GRB 050709 
6 Fox et al.

with the $z=0.16$ galaxy. Thus we show here, for the first time, that some SHBs arise in low-redshift star-forming galaxies.

The morphology of the host galaxy is irregular, typical of star-forming galaxies. We fit the radial light profile and find that it is well described by an exponential disk with scale length $r_{e}=0.75^{\prime \prime}$. The afterglow is situated 1.38 arcsec or $1.8 r_{e}$ from the brightest central region of the galaxy, corresponding to a physical offset of $3.8 \mathrm{kpc}$. From the detected emission lines we derive a star formation rate of $0.2 M_{\odot} \mathrm{yr}^{-1}$ (a lower limit after allowing for extinction). By comparison, long-duration GRBs are found exclusively in late-type (star-forming) host galaxies, ${ }^{17}$ and with a somewhat smaller median offset ${ }^{17}$ of $1.0 r_{e}$.

\section{Burst and afterglow energetics}

At a redshift of $z=0.16$, the isotropic-equivalent energy release in $\gamma$-rays ${ }^{10}$ over the $25-2000 \mathrm{keV}$ band is $E_{\gamma, \text { iso }}=6.9_{-0.5}^{+1.0} \times 10^{49} \mathrm{erg}$ and the peak luminosity is $L_{p}=(1.1 \pm$ $0.5) \times 10^{51} \mathrm{erg} \mathrm{s}^{-1}$ (here and throughout this paper, we adopt a flat cosmology with $H_{0}=71 \mathrm{~km} \mathrm{~s}^{-1} \mathrm{Mpc}^{-1}, \Omega_{\mathrm{M}}=0.27$ and $\left.\Omega_{\Lambda}=0.73\right)$. The burst of $\gamma$-rays is followed by an X-ray flare, detected by HETE from $25 \mathrm{~s}$ to $130 \mathrm{~s}$ after the burst. ${ }^{10}$ The fluence of this X-ray flare is twice as much as that of the $\gamma$-ray burst itself. Thus, the total isotropic energy release in the first few hundred seconds is $E_{\text {iso }} \sim 10^{50} \mathrm{erg}$, two orders of magnitude smaller than that seen in long-duration bursts. ${ }^{18}$

Figure 3 presents our observations of the GRB 050709 afterglow. The steepening powerlaw decay seen in our HST observations, a familiar feature of long-duration GRB light curves, is usually explained as arising from the collimated or jet-like nature of the ejecta. ${ }^{19}$ This is the first observation of such a light-curve "jet break" for a short-hard burst, although the steep $(\alpha \approx-2)$ decay of the GRB 050724 afterglow is suggestive. ${ }^{20}$ The epoch of steepening, $t_{b} \approx 10 \mathrm{~d}$, can be related to the opening angle of the jet, $\theta_{j}$ (in radians), as follows ${ }^{18}$ :

$$
\theta_{j}=0.076\left(\frac{t_{b}}{1 \text { day }}\right)^{3 / 8}\left(\frac{1+z}{2}\right)^{-3 / 8}\left(\frac{\eta_{\gamma}}{0.2}\right)^{1 / 8}\left(\frac{n}{1 \mathrm{~cm}^{-3}}\right)^{1 / 8}\left[\frac{E_{\gamma, \text { iso }}}{10^{53} \mathrm{erg}}\right]^{-1 / 8} \text { radian; }
$$

here $n$ is the circumburst particle density and $\eta_{\gamma}$ is the ratio of the radiated energy to the energy in relativistic ejecta. Setting $E_{\gamma, \text { iso }}=10^{50} \mathrm{erg}$ and $n=10^{-2} \mathrm{~cm}^{-3}$ we obtain $\theta_{j}=0.25$ 
and a beaming fraction, $f_{b}=1-\cos (\theta) \approx 0.03$. With this value of $f_{b}$, the energy released in the first few hundred seconds is $3 \times 10^{48} \mathrm{erg}$ - two orders of magnitude less than that inferred for long-duration GRBs. ${ }^{18}$

We interpret the fading optical and X-ray emission to be the afterglow of GRB 050709 . The afterglow phenomenon has been studied both observationally and theoretically in the context of long-duration GRBs. ${ }^{1}$ The emission arises from ambient material shocked by the relativistic ejecta. The broadband spectral index between the optical and X-ray bands at late times (Chandra and HST observations) is $\beta_{\mathrm{ox}}=-1.1$; since the temporal power-law index is $\alpha_{\mathrm{o}}=-1.25$ in the optical prior to the jet break, the electron power-law index is derived to be $\mathrm{p}=2.7$, and we find that the synchrotron cooling frequency is between the optical and X-ray bands at this time.

The isotropic-equivalent $\mathrm{X}$-ray luminosity $L_{\mathrm{X} \text {,iso }}$ of the afterglow at a fixed time after the burst serves as a useful proxy ${ }^{21}$ for the kinetic energy of the ejecta in the afterglow phase. Extrapolating back from the first Chandra flux to a fiducial time of $10 \mathrm{hr}$ post-burst, assuming a $t^{-1.3}$ decay (Fig. 3), we find $L_{\mathrm{X} \text {,iso }} \sim 2 \times 10^{42} \mathrm{erg} \mathrm{s}^{-1}$, at least three orders of magnitude smaller than is typical for the afterglows of long-duration GRBs. ${ }^{22}$

\section{Limits on an associated supernova}

At $z=0.16$, the distance modulus of GRB 050709 is $(m-M)=39.4$ mag. Our HST data (Fig. 1) are consistent with a pure afterglow evolution, and show no evidence for a supernova. Since we detect the afterglow in optical light, we are in the unique position (for SHBs) of being able to exclude any role for extinction in suppressing the light of an associated supernova. We estimate our sensitivity limit for an SN bump as the faintest magnitude we observe, $m_{\mathrm{SN}}>27.5 \mathrm{mag}$. Converting to absolute magnitudes and applying a $k$-correction (from F814W AB to Vega magnitudes) of $-0.12 \mathrm{mag}$, we estimate $M_{R}>-12.0 \mathrm{mag}$ for any associated SN at an age of $16 \mathrm{~d}$. This limit is fainter than any supernova yet detected in the nearby Universe.

Numerical studies of $\mathrm{SHBs}^{23}$ predict a modest associated nova-type event, much fainter 
8 Fox et al.

than the average supernova. Quantitative predictions for the ejecta mass, speed, and composition span an extremely broad range, however, so that the situation is ripe for observational inputs.

In the absence of heat input after the explosion, adiabatic expansion of any sub-relativistic ejecta results in very rapid fading. ${ }^{24,25}$ Continued heat input might by provided by the decay of radioactive nuclei (including nickel), ${ }^{24}$ decay of free neutrons, ${ }^{25}$ or a long-lived central engine. ${ }^{25}$ Any such heat input will be reprocessed to lower energies - mainly via electron Thompson scattering - provided the ejecta are dense enough. The high sensitivity of current facilities makes optical wavelengths the band of choice for these searches.

Our observations constrain the kinetic energy of slow ejecta in these models ${ }^{25}$ to be less than $10^{49} \mathrm{erg}$, provided that the ejecta velocity $v \lesssim 0.02 c$. The current limit arises entirely from the first-epoch HST data. Sensitive optical data taken at earlier times would have provided stronger constraints and for higher ejecta speeds.

An alternative source of heat could be luminosity from a long-lived central engine. The HST observations constrain this luminosity to be $L \lesssim 10^{41} \mathrm{erg} \mathrm{s}^{-1}$. We remark that the X-ray flare in the second epoch Chandra data has a similar luminosity.

\section{Properties of short-hard bursts}

The offsets of GRB 050709 and GBB 050724 from their proposed host galaxies are small enough that their associations can be considered secure. These two associations, in turn, strengthen the case for identification of GRB 050509B (localized to 9.3-arcsec radius ${ }^{6}$ ) with the redshift $z=0.225$ galaxy $^{5}$ and galaxy cluster ${ }^{6}$ that have been proposed to host this burst. We can thus set the physical scale for the energetics of all four bursts. Table 2 presents the properties of these SHBs, along with some properties of their host galaxies. Figure 4 places these bursts - the only known SHB afterglows - in the context of the set of long-duration GRBs with known redshifts.

In Table 2 we display the peak luminosities of the four SHBs, extrapolated to the full 
BATSE band for comparison with results from that experiment. All four values are approximately $L_{\gamma, \text { peak }} \sim 10^{50} \mathrm{erg} \mathrm{s}^{-1}$. The similarity of the redshifts and peak luminosities of the SHBs suggests that they arise from a single source population.

Next, from Figure 4 we see, relative to the long duration GRBs, SHBs are located at lower redshift, emit less energy in $\gamma$-rays, and possess a less-energetic afterglow. This behaviour is broadly consistent with conclusions from earlier statistical studies. ${ }^{26,7,27,9}$

A closer distance scale for the SHBs is consistent with the value of $\left\langle V / V_{\max }\right\rangle \approx 0.4$ for BATSE SHBs, ${ }^{28,9}$ which is significantly higher than the BATSE value for long-duration GRBs. In particular, the BATSE SHB $\left\langle V / V_{\max }\right\rangle$ value is consistent with a spatially-uniform distribution of standard candles out to $z_{\max } \simeq 0.4^{29}$ and with peak luminosities of $L_{\gamma, \text { peak }} \approx$ $10^{50} \mathrm{erg} \mathrm{s}^{-1}$ as observed for these four bursts.

In Table 2 we also summarize selected properties of the four SHB host galaxies. The proposed host of GRB 050509B is a large elliptical galaxy at redshift $z=0.2248,{ }^{5}$ with luminosity $L \approx 1.5 L_{*}$ (ref. 30). The galaxy has little ongoing star formation, $<0.1 M_{\odot} \mathrm{yr}^{-1}$ (ref. 5). The elliptical host galaxy of GRB 050724 (which has also been localized to subarcsecond precision) is a luminous $\left(L=1.6 L_{*}\right)$ elliptical galaxy with star formation rate $<0.02 M_{\odot} \mathrm{yr}^{-1}($ ref. 20$)$.

In contrast, the morphology and spectrum of the host galaxy of GRB 050709 (Figure 1) indicates that the host is an irregular, late-type galaxy with a significant star formation rate, $\sim 0.2 M_{\odot} \mathrm{yr}^{-1}$, and a luminosity much smaller than the other SHB hosts, $L \approx 0.10 L_{*}$; thus the galaxy is forming roughly as many stars per unit stellar mass as the Milky Way.

The association of SHBs with both star-forming galaxies and early-type ellipticals is reminiscent of the diversity of host galaxies of type Ia supernovae ( $\mathrm{SNe}$ ). As with the type Ia SNe, this dichotomy may indicate a class of progenitors with an extremely wide range of lifetimes between formation and explosion, with some systems living for many gigayears. However, even though type Ia SNe occur in elliptical galaxies, the rate of such events is higher in late-type, star-forming galaxies, and the majority of type Ia events in the local Universe occur in late-type blue galaxies. ${ }^{31}$ The trend emerging for the SHBs, in which 
10 Fox et al.

the "majority" of events (here perhaps, 3 of 4) occur in elliptical galaxies, could indicate that the progenitor systems are even longer-lived than those of Ia supernovae.

\section{The nature of the short-hard bursts}

Our observations of GRB 050709 support the view - until recently founded only on the basis of their prompt $\gamma$-ray emissions - that the SHBs are a different population from the long-duration GRBs (Fig. 4). They are lower-energy explosions, with a correspondingly lessenergetic relativistic blastwave, and they are found at significantly closer distances. At the same time, the similarity of the SHB redshifts and peak luminosities to one other (Table 2) strongly suggests a common origin for these events.

We find that SHBs are distinctly weaker explosions than long-duration GRBs. The lower energies that we infer from afterglow observations of GRB 050709 and GRB 050724 are consistent with the merger of a compact object binary, as seen in numerical simulations. Moreover, the two best-studied events show strong collimation; thus the true rate of SHBs is 30 to 100 times the observed rate.

The long-duration GRBs have been definitively associated with the deaths of massive stars. $^{32-35}$ Two properties of the known SHBs argue against such an association: first, the fact that GRB 050724 occurred in an elliptical galaxy without active star formation, ${ }^{20}$ and that GRB 050509B likely occurred in an elliptical as well; ${ }^{5}$ and second, that GRB $050509 B^{4,36}$ and GRB 050709 lack associated supernovae, a common feature of $z<1$ long-duration GRBs, to very deep limits. Separately, the presence of SHBs among old stellar populations in elliptical galaxies argues against a magnetar origin, or a form of short-lived compact binary.

The locations of the SHBs with respect to their host galaxies are compatible with the kicks delivered to neutron star binary systems at birth. ${ }^{37}$ Their occurrence in both starforming (late-type) and non-star-forming (early-type) galaxies suggests that there may be a substantial range of lifetimes for the progenitor systems, perhaps extending to many gigayears.

In all respects, the emerging picture of SHB properties is consistent with an origin in the coalescence events of neutron star-neutron star or neutron star-black hole binary systems. 
The stage is now set for detailed studies of these exotic cosmic explosions, the most exciting of which would be the detection of their associated bursts of gravitational waves.

Received 13 August 2018; Accepted draft.

1. Zhang, B. \& Meszaros, P. Gamma-ray bursts: Progress, problems and prospects. Int. J. Mod. Phys. A19, 2385-2472 (2004).

2. Kouveliotou, C., Meegan, C. A., Fishman, G. J., Bhat, N. P., Briggs, M. S. et al. Identification of two classes of gamma-ray bursts. Astrophys. J. 413, L101-L104 August 1993.

3. Hurley, K., Berger, E., Castro-Tirado, A., Castro Cerón, J. M., Cline, T. et al. Afterglow Upper Limits for Four Short-Duration, Hard Spectrum Gamma-Ray Bursts. Astrophys. J. 567, 447-453 (2002).

4. Kulkarni, S. R. et al. Supernova and low-velocity ejecta constraints for the short-hard $\gamma$-ray burst GRB 050509B. submitted to Nature (2005).

5. Bloom, J. S., Prochaska, J. X., Pooley, D., Blake, C. W., Foley, R. J. et al. Closing in on a Short-Hard Burst Progenitor: Constraints from Early-Time Optical Imaging and Spectroscopy of a Possible Host Galaxy of GRB 050509b. submitted to Astrophys. J. May 2005.

6. Gehrels, N., Barbier, L., Barthelmy, S. D., Blustin, A., Burrows, D. N. et al. The first localization of a short gamma-ray burst by Swift. Nature, submitted (2005).

7. Schmidt, M. Luminosities and Space Densities of Short Gamma-Ray Bursts. Astrophys. J. 559, L79-L82 October 2001.

8. Eichler, D., Livio, M., Piran, T. \& Schramm, D. N. Nucleosynthesis, neutrino bursts and gamma-rays from coalescing neutron stars. Nature 340, 126-128 July 1989.

9. Guetta, D. \& Piran, T. The luminosity and redshift distributions of short-duration GRBs. Astr. Astrophys. 435, 421-426 May 2005.

10. Villasenor, J., Lamb, D. Q., Ricker, G. R., Atteia, J.-L., Kawai, N. et al. HETE-2 Discovery of the short $\gamma$-ray burst GRB 050709. Nature, submitted (2005). 
12 Fox et al.

11. Garmire, G. P., Bautz, M. W., Ford, P. G., Nousek, J. A. \& Ricker, G. R. in X-Ray and GammaRay Telescopes and Instruments for Astronomy. Edited by Joachim E. Truemper, Harvey D. Tananbaum. Proceedings of the SPIE, Volume 4851, pp. 28-44 (2003). 28-44 March 2003.

12. Condon, J. J., Cotton, W. D., Greisen, E. W., Yin, Q. F., Perley, R. A. et al. The NRAO VLA Sky Survey. Astron. J. 115, 1693-1716 May 1998.

13. Fox, D. B., Frail, D. A., Cameron, P. B. \& Cenko, S. B. GRB050709: Candidate X-ray Afterglow. GRB Circular Network 3585, 1-1 (2004).

14. Burrows, D. N., Romano, P., Falcone, A., Kobayashi, S., Zhang, B. et al. Bright X-ray Flares in Gamma-Ray Burst Afterglows. Science 150, 1-5 July 2005.

15. Hjorth, J., Watson, D., Fynbo, J. P. U. et al. The optical afterglow of the short $\gamma$-ray burst GRB 05709. Nature in press (2005).

16. Sirianni, M., Jee, M. J., Benitez, N., Blakeslee, J. P., Martel, A. R. et al. The Photometric Performance and Calibration of the HST Advanced Camera For Surveys. PASP (in press) July 2005.

17. Bloom, J. S., Kulkarni, S. R. \& Djorgovski, S. G. The Observed Offset Distribution of GammaRay Bursts from Their Host Galaxies: A Robust Clue to the Nature of the Progenitors. Astron. J. 123, 1111-1148 March 2002.

18. Frail, D. A., Kulkarni, S. R., Sari, R., Djorgovski, S. G., Bloom, J. S. et al. Beaming in GammaRay Bursts: Evidence for a Standard Energy Reservoir. Astrophys. J. 562, L55-L58 November 2001.

19. Rhoads, J. E. The Dynamics and Light Curves of Beamed Gamma-Ray Burst Afterglows. Astrophys. J. 525, 737-749 November 1999.

20. Berger, E., Price, P. A., Cenko, S. B., Gal-Yam, A., Soderberg, A. M. et al. A Merger Origin for Short Gamma-Ray Bursts Inferred from the Afterglow and Host Galaxy of GRB 050724. ArXiv.org , 1-15 August 2005. Submitted to Nature.

21. Kumar, P. The distribution of burst energy and shock parameters for gamma-ray bursts. Astrophys. J. 538, L125-L128 (2000). 
22. Berger, E., Kulkarni, S. R. \& Frail, D. A. A Standard Kinetic Energy Reservoir in Gamma-Ray Burst Afterglows. Astrophys. J. 590, 379-385 June 2003.

23. Janka, H.-T. \& Ruffert, M. in ASP Conf. Ser. 263: Stellar Collisions, Mergers and their Consequences $333-+2002)$.

24. Li, L. \& Paczyński, B. Transient Events from Neutron Star Mergers. Astrophys. J. 507, L59L62 November 1998.

25. Kulkarni, S. R. Modeling possible macronova associated with short hard bursts. in prep (2005).

26. Totani, T. Probing the Cosmic Star Formation History by the Brightness Distribution of Gamma-Ray Bursts. Astrophys. J. 511, 41-55 January 1999.

27. Balázs, L. G., Bagoly, Z., Horváth, I., Mészáros, A. \& Mészáros, P. On the difference between the short and long gamma-ray bursts. Astr. Astrophys. 401, 129-140 April 2003.

28. Katz, J. I. \& Canel, L. M. The Long and the Short of Gamma-Ray Bursts. Astrophys. J. 471, 915-+ November 1996.

29. Piran, T. in IAU Symp. 165: Compact Stars in Binaries 489-+ 1996).

30. Eisenstein, D. J., Hogg, D. W. \& Padmanabhan, N. GRB050509b, SDSS pre-burst observations. GRB Circular Network 3418, 1-+ (2005).

31. Mannucci, F., della Valle, M., Panagia, N., Cappellaro, E., Cresci, G. et al. The supernova rate per unit mass. Astr. Astrophys. 433, 807-814 April 2005.

32. Kulkarni, S. R., Frail, D. A., Wieringa, M. H., Ekers, R. D., Sadler, E. M. et al. Radio emission from the unusual supernova 1998bw and its association with the gamma-ray burst of 25 April 1998. Nature 395, 663-669 (1998).

33. Galama, T. J., Vreeswijk, P. M., van Paradijs, J., Kouveliotou, C., Augusteijn, T. et al. An unusual supernova in the error box of the gamma-ray burst of 25 April 1998. Nature 395, 670-672 (1998).

34. Hjorth, J., Sollerman, J., Møller, P., Fynbo, J. P. U., Woosley, S. E. et al. A very energetic supernova associated with the $\gamma$-ray burst of 29 March 2003. Nature 423, 847-850 June 2003. 
14 Fox et al.

35. Stanek, K. Z., Matheson, T., Garnavich, P. M., Martini, P., Berlind, P. et al. Spectroscopic Discovery of the Supernova 2003dh Associated with GRB 030329. Astrophys. J. 591, L17-L20 July 2003.

36. Hjorth, J., Sollerman, J., Gorosabel, J., Granot, J., Klose, S. et al. GRB 050509B: Constraints on short gamma-ray burst models. ApJL in press; astro-ph/0506123 June 2005.

37. Narayan, R., Paczynski, B. \& Piran, T. Gamma-ray bursts as the death throes of massive binary stars. Astrophys. J. 395, L83-L86 August 1992.

38. Morgan, A., Grupe, D., Gronwall, C., Racusin, J., Falcone, A. et al. GRB 050709: Swift UVOT and XRT observations. GCN Circular 3577 (2005).

39. Schlegel, D. J., Finkbeiner, D. P. \& Davis, M. Maps of Dust Infrared Emission for Use in Estimation of Reddening and Cosmic Microwave Background Radiation Foregrounds. Astrophys. J. 500, 525-553 June 1998.

40. Motohara, K., Iwamuro, F., Maihara, T., Oya, S., Tsukamoto, H. et al. CISCO: Cooled Infrared Spectrograph and Camera for OHS on the Subaru Telescope. Proc. Astr. Soc. Jap. 54, 315-325 April 2002.

41. Fruchter, A. S. \& Hook, R. N. Drizzle: A Method for the Linear Reconstruction of Undersampled Images. Publ. Astr. Soc. Pacific 114, 144-152 February 2002.

42. Covino, S., Antonelli, L. A., Romano, P., Palmer, D., Markwardt, C. et al. GRB 050724: a short-burst detected by Swift. GCN Circular 3665 (2005).

43. Fox, D. B. et al. Swift Discovery and Observation of the Short-Hard Burst GRB 050813. in prep (2005).

44. Gladders, M. et al. Discovery of a Galaxy Cluster Associated with the Short-Hard Burst GRB 050813. in prep (2005).

45. Peng, C. Y., Ho, L. C., Impey, C. D. \& Rix, H.-W. Detailed Structural Decomposition of Galaxy Images. Astron. J. 124, 266-293 July 2002.

46. Berger, E., Kulkarni, S. R., Pooley, G., Frail, D. A., McIntyre, V. et al. A common origin for cosmic explosions inferred from calorimetry of GRB030329. Nature 426, 154-157 November 2003. 
47. Friedman, A. S. \& Bloom, J. S. Toward a More Standardized Candle Using Gamma-Ray Burst Energetics and Spectra. Astrophys. J. 627, 1-25 July 2005.

48. Berger, E., Kulkarni, S. R., Fox, D. B., Soderberg, A. M., Harrison, F. A. et al. The Afterglows, Redshifts, and Properties of Swift Gamma-Ray Bursts. ArXiv.org astro-ph/0505107, 1-10 May 2005. Submitted to ApJ.

\section{Acknowledgements}

Our GRB research is supported in part by funds from NSF, NASA, the Australian Research Council, and the Ministry of Education, Science, Culture, Sports, and Technology in Japan. The VLA is operated by the National Radio Astronomy Observatory, a facility of the NSF operated under cooperative agreement by Associated Universities, Inc. The Gemini Observatory is operated by the Association of Universities for Research in Astronomy (AURA), Inc., under a cooperative agreement with the NSF on behalf of the Gemini partnership. This work is based in part on data collected at Subaru Telescope, which is operated by the National Astronomical Observatory of Japan. 


\begin{tabular}{lllllll}
\hline \hline UT Date & UT & $\Delta T$ & Facility & Band & Exp & Flux \\
\hline \hline 11-Jul-2005 & $04: 20$ & $1.24 \mathrm{~d}$ & Swope-40 & $i^{\prime}$ & $3 \times 600 \mathrm{~s}$ & $>20.5 \mathrm{mag}$ \\
11-Jul-2005 & $10: 19$ & $1.49 \mathrm{~d}$ & Swope-40 & $i^{\prime}$ & $3 \times 600 \mathrm{~s}$ &.. \\
13-Jul-2005 & $08: 33$ & $3.41 \mathrm{~d}$ & Du Pont-100 & $R$ & $3 \times 600 \mathrm{~s}$ & $(21.05 \pm 0.15 \mathrm{mag})$ \\
15-Jul-2005 & $14: 06$ & $5.6 \mathrm{~d}$ & Subaru & $K^{\prime}$ & $270 \times 20 \mathrm{~s}$ & $22.1 \pm 0.7 \mathrm{mag}$ \\
26-Jul-2005 & $13: 19$ & $16.6 \mathrm{~d}$ & Subaru & $K^{\prime}$ & $360 \times 20 \mathrm{~s}$ & $(19.2 \pm 0.1)$ \\
\hline 11-Jul-2005 & $12: 14$ & $1.6 \mathrm{~d}$ & VLA & $8.46 \mathrm{GHz}$ & $6660 \mathrm{~s}$ & $<76.6 \mu \mathrm{Jy}$ \\
12-Jul-2005 & $09: 36$ & $2.5 \mathrm{~d}$ & VLA & $8.46 \mathrm{GHz}$ & $1265 \mathrm{~s}$ & $<75.8 \mu \mathrm{Jy}$ \\
14-Jul-2005 & $11: 31$ & $4.5 \mathrm{~d}$ & VLA & $8.46 \mathrm{GHz}$ & $6055 \mathrm{~s}$ & $<49.4 \mu \mathrm{Jy}$ \\
17-Jul-2005 & $10: 48$ & $7.5 \mathrm{~d}$ & VLA & $8.46 \mathrm{GHz}$ & $6490 \mathrm{~s}$ & $<26.6 \mu \mathrm{Jy}$ \\
\hline 09-Jul-2005 & $22: 38$ & $100 \mathrm{~s}$ & HETE WXM & $5 \mathrm{keV}$ & $10 \mathrm{~s}$ & $0.80 \pm 0.14 \mathrm{mJy}$ \\
\hline 12-Jul-2005 & $11: 15$ & $2.5 \mathrm{~d}$ & Chandra & $5 \mathrm{keV}$ & $38.4 \mathrm{ks}$ & $0.15 \pm 0.02 \mathrm{nJy}$ \\
25-Jul-2005 & $21: 47$ & $16.0 \mathrm{~d}$ & Chandra & $5 \mathrm{keV}$ & $6.1 \mathrm{ks}$ & $0.18 \pm 0.06 \mathrm{nJy}$ \\
26-Jul-2005 & $00: 18$ & $16.1 \mathrm{~d}$ & Chandra & $5 \mathrm{keV}$ & $12.1 \mathrm{ks}$ & $0.015+0.027 \mathrm{nJy}$ \\
\hline 11-Jul-2005 & $13: 07$ & $1.6 \mathrm{~d}$ & Swift & $5 \mathrm{keV}$ & $15.2 \mathrm{ks}$ & $0.26 \pm 0.14 \mathrm{nJy}$ \\
12-Jul-2005 & $10: 13$ & $2.4 \mathrm{~d}$ & Swift & $5 \mathrm{keV}$ & $5.0 \mathrm{ks}$ & $<0.2 \mathrm{nJy}$ \\
13-Jul-2005 & $02: 32$ & $3.2 \mathrm{~d}$ & Swift & $5 \mathrm{keV}$ & $6.4 \mathrm{ks}$ & $<0.1 \mathrm{nJy}$ \\
14-Jul-2005 & $05: 26$ & $4.3 \mathrm{~d}$ & Swift & $5 \mathrm{keV}$ & $2.1 \mathrm{ks}$ & $<0.8 \mathrm{nJy}$ \\
\hline 15-Jul-2005 & $13: 49$ & $5.6 \mathrm{~d}$ & HST & F814W & $6360 \mathrm{~s}$ & $25.08 \pm 0.02 \mathrm{mag}$ \\
19-Jul-2005 & $17: 11$ & $9.8 \mathrm{~d}$ & HST & F814W & $6360 \mathrm{~s}$ & $25.84 \pm 0.05 \mathrm{mag}$ \\
28-Jul-2005 & $13: 48$ & $18.6 \mathrm{~d}$ & HST & F814W & $6360 \mathrm{~s}$ & $27.81 \pm 0.27 \mathrm{mag}$ \\
13-Aug-2005 & $15: 17$ & $34.7 \mathrm{~d}$ & HST & F814W & $6360 \mathrm{~s}$ & $>28.1 \mathrm{mag}$ \\
\hline \hline
\end{tabular}

Table 1. Observations of the afterglow of GRB 050709

We carried out all observations summarized in this table except those of HETE (from ref. 10) and Swift (reported in ref. 38). The HETE point represents the average flux in the "soft flare" that occurs $100 \mathrm{~s}$ after the main burst. We have made an independent reduction of the Swift XRT data. Optical \& Near Infrared. The optical and near infrared fluxes do not contain a correction for Galactic extinction which is expected to be rather small in this direction ${ }^{39}: E(B-V)=0.012$ mag. Near-infrared observations were made with the Cool Infrared Spectrograph and Camera for $\mathrm{OH}$ Suppression $^{40}$ (CISCO). No flux is reported for the Swope-40 second epoch since the image was subtracted from the first epoch in order to search for the afterglow. Host fluxes in the table are given in parentheses.

Radio. The VLA data were taken in standard continuum mode with a bandwidth of $2 \times 50$ $\mathrm{MHz}$ centered in the $8.46-\mathrm{GHz}$ band. We used $3 \mathrm{C} 48$ for flux calibration and phase referencing 
was performed against calibrator J2257-364. Data were reduced using standard packages within the Astronomical Image Processing System (AIPS). Within the HETE error circle we find a single source with constant flux $(644 \pm 24 \mu \mathrm{Jy})$ at coordinates $\alpha=23: 01: 32.1 \pm 0.003, \delta=-38: 59: 26.8 \pm 0.1$ (J2000). This source is coincident with the cataloged extended NVSS source and resolved Chandra source. Upper limits are quoted at $2 \sigma$ or $95.5 \%$ confidence.

Chandra X-ray Observatory. Our basic data reduction procedures are described in the text. We use a custom pipeline composed of CIAO 3.2.1 tools to run a full "wavdetect" analysis on images constructed from the $0.3-2.0 \mathrm{keV}, 2.0-8.0 \mathrm{keV}$, and $0.3-8.0 \mathrm{keV}$ bands separately, and then merge the resulting source catalogs. For spectral fits, photons are extracted from a 1.5"-radius aperture, and fits are made using XSPEC 12.0. The second observation has been divided into "flaring" and "quiescent" intervals; see text for details.

Swift X-ray Telescope. The photometry was done using a circular region of radius 0.8 arcmin centered on the X-ray afterglow position. The background was estimated from the entire XRT image. The detection confidence level from Poisson statistics is $2.3 \sigma$ for the first observation; the remaining observations provide only upper limits (quoted at $2 \sigma$ or $95.5 \%$-confidence). Photon count limits are converted to flux densities using our spectral fit to the first-epoch Chandra data; see text for details.

Hubble Space Telescope. Data were obtained with the ACS instrument aboard HST. ${ }^{16}$ Each epoch consisted of a series of exposures in the F814W (I-band) filter with a total integration time of $6360 \mathrm{~s}$. The images were processed using Archive "on-the-fly" processing, drizzled ${ }^{41}$ to the native pixel scale of 0.05 arcsecond, and combined. We subtracted the fourth-epoch image from each previous epoch and performed photometry using a $0.15^{\prime \prime}$ (3-pixel) aperture. The magnitudes, quoted in the "AB" system, are corrected for finite aperture (0.32 mag) and imperfect charge transfer efficiency (0.01 mag). Errors are estimated by photometering multiple background regions in the subtracted images using the same aperture. The limit on afterglow flux in the fourth epoch is derived by subtracting point sources of decreasing flux from the image until a flux deficit at the afterglow position is no longer readily discernible. 


\begin{tabular}{lcccc}
\hline Property & GRB 050509B & GRB 050709 & GRB 050724 & GRB 050813 \\
\hline Redshift & 0.225 & 0.160 & 0.258 & 0.722 \\
$T_{90}$ & $40 \pm 4 \mathrm{~ms}$ & $70 \pm 10 \mathrm{~ms}$ & $3 \pm 1 \mathrm{~s}$ & $0.6 \pm 0.1 \mathrm{~s}$ \\
Fluence $\left(\mathrm{erg} \mathrm{cm}^{-2}\right)$ & $9.5 \times 10^{-9}$ & $2.9 \times 10^{-7}$ & $6.3 \times 10^{-7}$ & $1.2 \times 10^{-7}$ \\
Fluence band & $15-350 \mathrm{keV}$ & $30-400 \mathrm{keV}$ & $15-350 \mathrm{keV}$ & $15-350 \mathrm{keV}$ \\
$E_{\gamma, \text { iso }}(\mathrm{erg})$ & $4.5 \times 10^{48}$ & $6.9 \times 10^{49}$ & $4.0 \times 10^{50}$ & $6.5 \times 10^{50}$ \\
$L_{\gamma, \text { peak }}\left(\mathrm{erg} \mathrm{s}^{-1}\right)$ & $1.4 \times 10^{50}$ & $1.1 \times 10^{51}$ & $1.7 \times 10^{50}$ & $1.9 \times 10^{51}$ \\
$L_{\mathrm{X}}\left(\mathrm{erg} \mathrm{s}^{-1}\right)$ & $<7 \times 10^{41}$ & $3 \times 10^{42}$ & $8 \times 10^{43}$ & $9 \times 10^{43}$ \\
$f_{b}$ & - & 0.03 & 0.01 & - \\
$E_{\gamma}(\mathrm{erg})$ & $<4.5 \times 10^{48}$ & $2.1 \times 10^{48}$ & $4.0 \times 10^{48}$ & $<6.5 \times 10^{50}$ \\
Host $L / L_{*}$ & 1.5 & 0.10 & 1.6 & - \\
Host SFR $\left(M_{\odot} \mathrm{yr}^{-1}\right)$ & $<0.1$ & 0.2 & $<0.03$ & - \\
Offset $(\mathrm{kpc})$ & $44_{-23}^{+12}$ & 3.8 & 2.6 & - \\
Offset $\left(r / r_{e}\right)$ & $13_{-7}^{+3}$ & 1.8 & 0.4 & - \\
SN Limit, $M_{R}(\mathrm{mag})$ & $>-13.0$ & $>-12.0$ & - & - \\
\hline
\end{tabular}

Table 2. Physical properties of short-hard bursts and their host galaxies

In order, we show: the burst redshift; the duration of the 90\%-inclusive interval of high-energy emission $\left(T_{90}\right)$; the measured burst fluence and corresponding energy bandpass; the peak burst luminosity; the isotropic-equivalent $\gamma$-ray burst energy; the isotropic-equivalent luminosity in X-ray at $10 \mathrm{~h}$ post-burst; the beaming fraction, calculated from the jet collimation angle $\theta_{j}$ as $f_{b}=$ $1-\cos \left(\theta_{j}\right)$; the $\gamma$ ray energy release corrected for beaming fraction, $f_{b} E_{\gamma, \text { iso }}$; the host galaxy luminosity; the host star-formation rate; the burst offset from its host, in physical units and referred to the scale length of the host galaxy's light profile; and the absolute magnitude limit on any associated supernova. Blank entries ("-") are unconstrained by the data at present; values without uncertainties are known to roughly $20 \%$ precision. References: GRB 050509B, refs. 6, 5 and 4; GRB 050709, ref. 10 and this work; GRB 050724, refs. 42 and 20; GRB 050813, refs. 43 and 44. The values of $E_{\gamma \text {,iso }}$ and $L_{\gamma, \text { peak }}$ have been increased by a factor of 4 , representing a mean correction from the BATSE sample for converting these observed fluences to the 25-2000 keV band. 

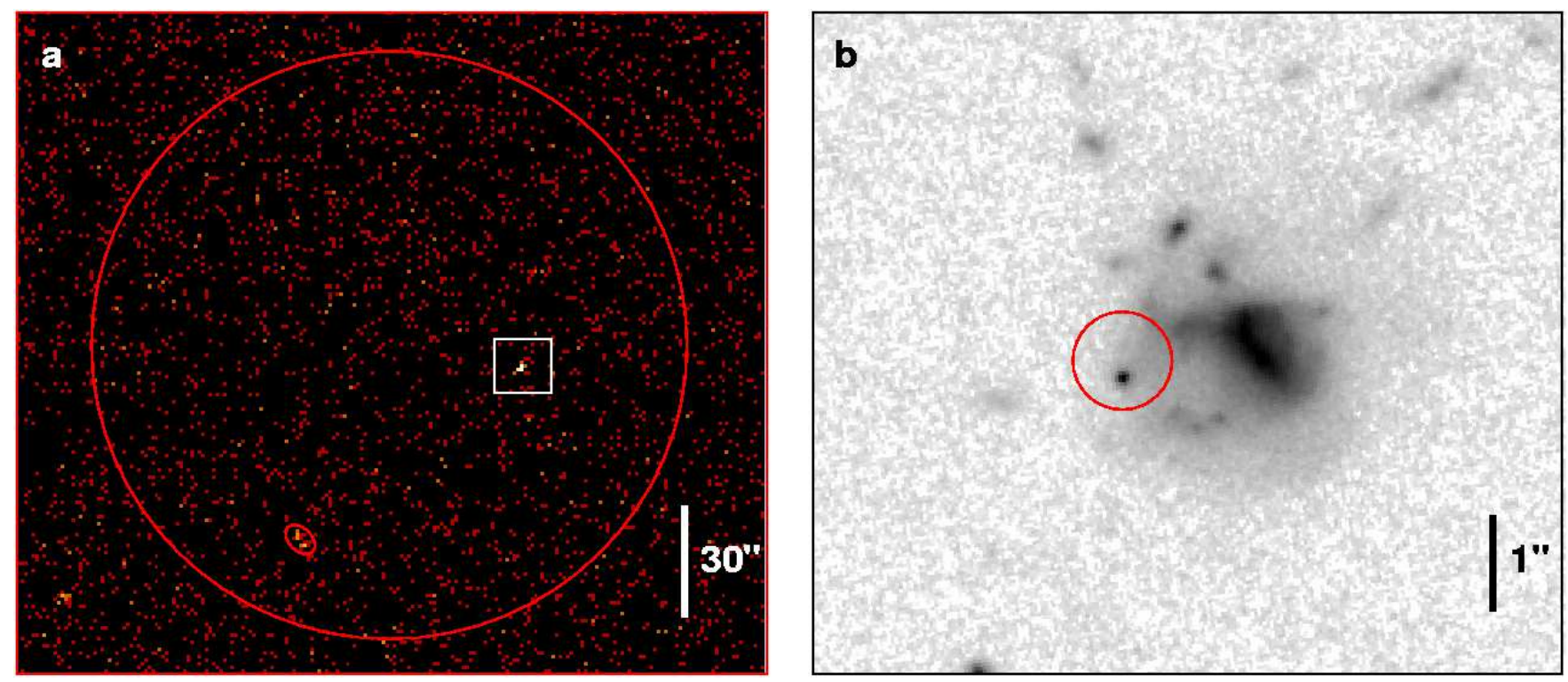

Figure 1. HST and Chandra X-ray Observatory images of the afterglow and environs of GRB 050709

(a) The Chandra (0.3-8.0 keV) image of the field from our observation of 2005 July 12.5 UT. The large circle is the HETE localization region, 81 arcsec in radius; north is up, east is to the left, and the scale of the image is indicated. A red ellipse indicates the faint X-ray source which we identify with an NVSS catalog object; the bright point source in the boxed region is the afterglow of GRB 050709. (b) Close-up of the region surrounding the X-ray afterglow, in a coaddition of all our HST data; the red circle is the Chandra localization region, 0.5 arcsec in radius. A point source is visible within this region; the source is observed to fade over the course of our HST observations, and we identify it as the optical afterglow of GRB 050709. The irregular galaxy to its west is the proposed $z=0.16$ host galaxy. We use the GALFIT software ${ }^{45}$ to fit the radial surface brightness distribution of the host galaxy, with the Sersic concentration parameter, $n$, and the effective radius, $r_{e}$, as free parameters. We find a best-fit solution ( $\chi 2 \approx 3$ per degree of freedom) with $n=1.1$ and $r_{e}=0.75^{\prime \prime}$. At $z=0.16$ the afterglow's 1.38-arcsec offset from the brightest region of this galaxy corresponds to $3.8 \mathrm{kpc}$ in projection, or $1.8 r_{e}$. 


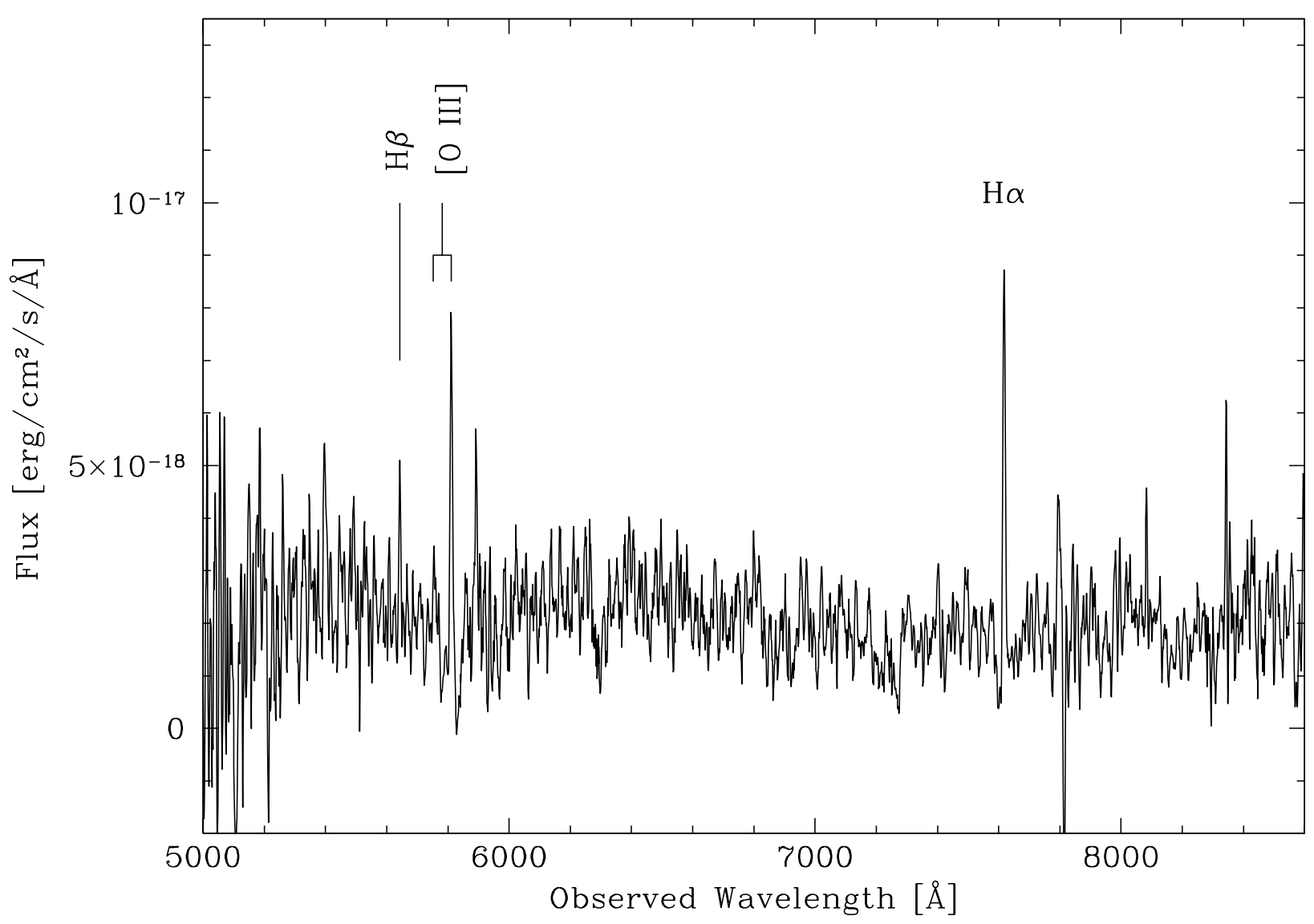

Figure 2. Spectrum of the host galaxy of GRB 050709.

These observations were taken with GMOS on the Gemini North telescope under poor seeing and non-photometric conditions. We obtained a total integration of $3,303 \mathrm{~s}$ in four exposures before closing due to the onset of twilight. The spectra were processed with the GMOS data reduction package in IRAF, sky-subtracted, combined and extracted, and smoothed with a $7 \AA$ boxcar. Flux calibration was performed using an observation of Hiltner 600 observed with a similar instrument setup in a previous lunation; hence the flux scale shown is indicative rather than absolute. Three emission lines are readily apparent $(\mathrm{H} \alpha,[\mathrm{OIII}] \lambda 5007$ and $\mathrm{H} \beta)$ yielding a mean redshift of $z=$ $0.160 \pm 0.001$. The observed $\mathrm{H} \alpha / \mathrm{H} \beta$ flux ratio of 3.1 indicates that the global galaxy extinction is small $\left(A_{V}=0.1 \mathrm{mag}\right)$. 


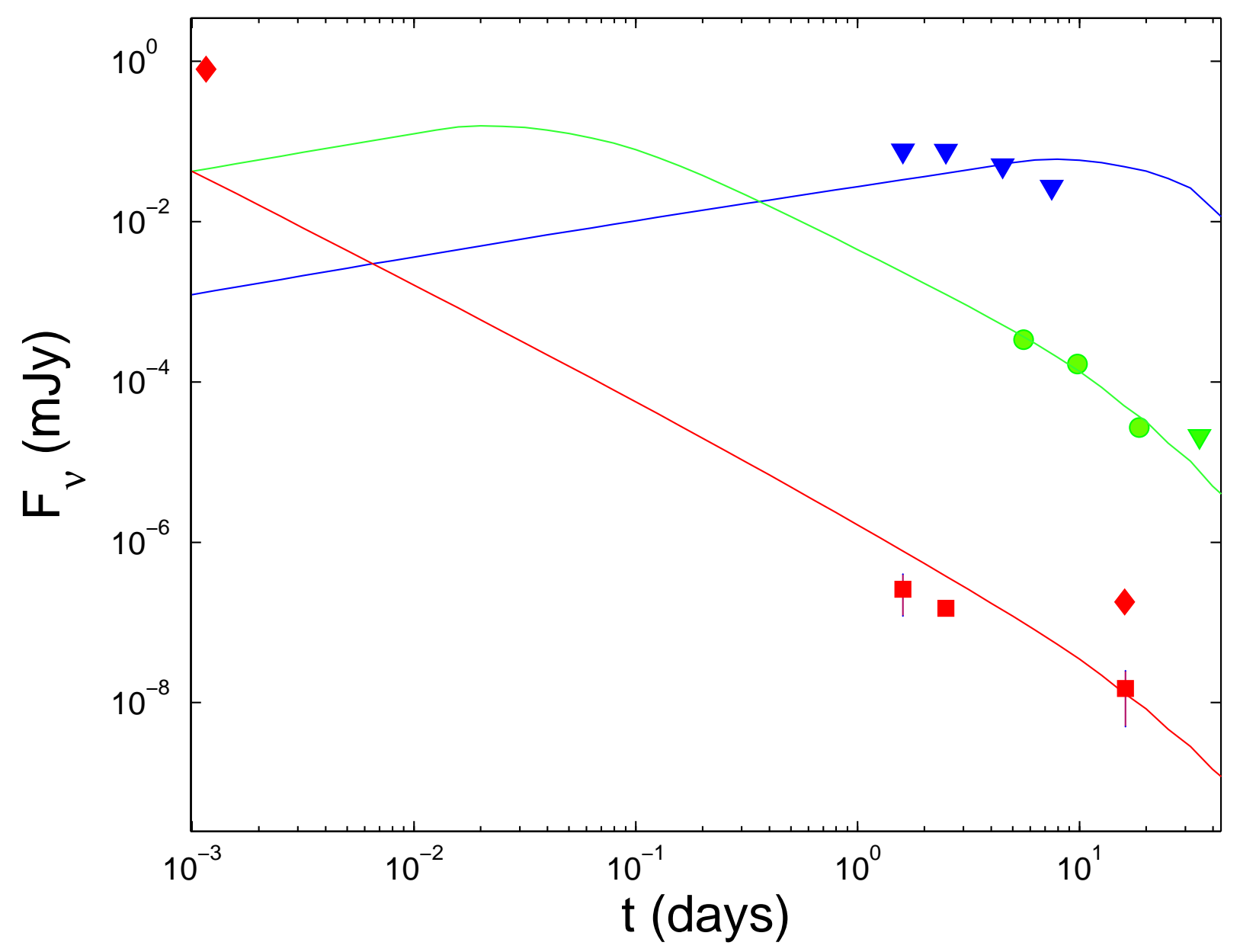

Figure 3. Observations of the GRB 050709 afterglow and illustrative models.

The X-ray (red), optical (green) and radio (blue) data taken from Table 1. These multi-wavelength observations can be marginally accommodated within a standard external-shock afterglow model. The best fit parameters are $E=5 \times 10^{48} \mathrm{erg}, \epsilon_{e}=\epsilon_{B}=1 / 3, n=0.01, p=2.5$ and $\theta_{j}=0.35$. However even this best fit predicts a radio flux which is slightly higher (a factor of 2) than our upper limits and requires an uncommonly high value for $\epsilon_{B}$. The latter is a concern because we do not expect the microphysics of the afterglow to differ between the short- and long-duration burst populations, although one cannot exclude this possibility. The early HETE X-ray observation and the late Chandra X-ray flare cannot be explained by the standard external shock afterglow model, both are too bright. The early point can be a flare resulting from an energy injection to the external shock or a long lasting activity of the source, as seen recently by the Swift/XRT in a long burst. ${ }^{14}$ 
The late flare however is a unique phenomenon to short bursts that was not observed so far in any long burst. The duration of the flare $(\sim 0.1$ day) corresponds to an emitting region at a size of $10^{14} \mathrm{~cm} 16$ days after the burst. At this time the external shock is mildly relativistic and its width is $\sim 10^{16} \mathrm{~cm}$. The (isotropic equivalent) energy emitted in this flare is $\sim 10^{45} \mathrm{erg}$ in the X-ray alone. These different length scales, together with brightness of the flare, exclude the possibility that the flare is produced in a simple external shock and indicates a process that was not observed so far in long bursts. Most probably, this process involves an activity of the source at $t \sim 16$ days which is $10^{7}$ times the duration of the burst. Combined with the unique X-ray light curve of the short-hard burst GRB 050724 (showing an early very steep decay and a later brightening) these observations suggest that one should keep an open mind for the possibility that SHB afterglows are a drastically different phenomenon than afterglows of long GRBs. 


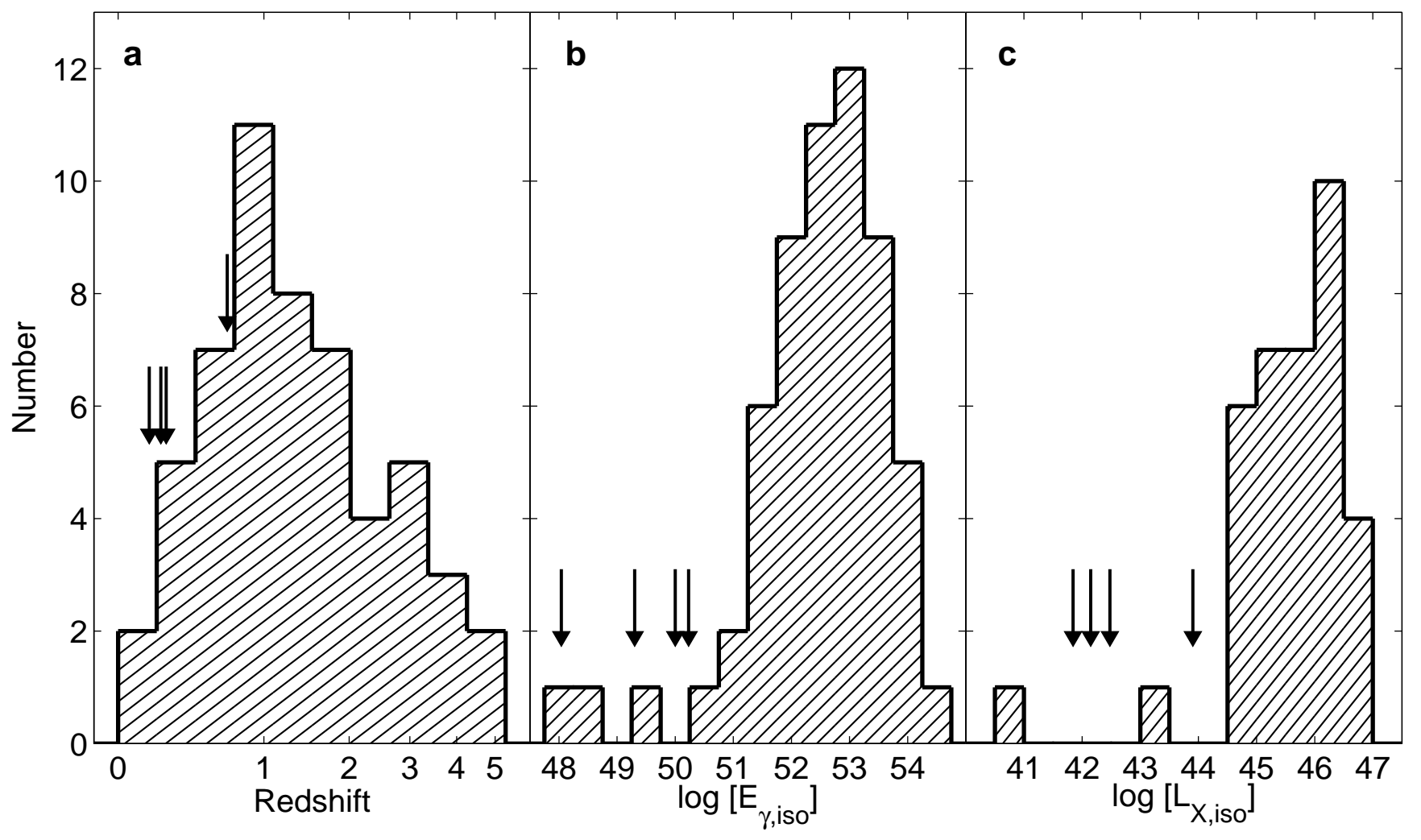

Figure 4. Physical properties of the afterglows of long-duration GRBs (histograms) and SHBs (arrows).

a-c, For GRBs with known redshifts, their distribution in redshift (a), isotropic-equivalent gammaray luminosity $\left(\log \left[E_{\gamma, \text { iso }}\right], \mathrm{b}\right)$, and isotropic-equivalent X-ray luminosity at 10 hours after the burst $\left(\log \left[L_{\mathrm{X}, \text { iso }}\right], \mathrm{c}\right)$. Arrows indicate the values of these properties for the four SHBs with afterglow detections, GRB 050509B, GRB 050709, GRB 050724, and GRB 050813 (as they are ordered consistently, from lowest to highest, in each panel). The contrast between the physical properties of the two burst populations is dramatic. GRB properties are from refs $22,46,47$, and 48 . See Table 2 for SHB references. 